\title{
A NEW FRAMEWORK FOR SOLVING DYNAMIC SCHEDULING GAMES
}

\author{
Santiago Zazo , Sergio Valcarcel, Matilde Sánchez-Fernández , Javier Zazo
}

\begin{abstract}
Optimum scheduling is a key objective in many communications systems where different users have to share a common resource. Typically, centralized implementations are capable of guaranteeing certain fairness. In our approach, we follow a different path modeling the scheduling process as a dynamic infinite horizon discrete-time game. This formulation allows us to include any kind of dynamics and distributed implementations. Despite, these games are very difficult to solve, we are able to show that they are in fact dynamic potential games equivalent to a non-stationary multivariate optimum control problem. The dynamic control problem is solved via an augmented Bellman equation including time as an extra state.
\end{abstract}

Index Terms - Optimum Scheduling, Fairness, Dynamic Potential Games, Augmented Bellman Equation

\section{INTRODUCTION}

Radio resources are usually of restricted use in many communication systems. Thus, letting several users make use simultaneously of a single resource in a wireless communication system implies in many cases limiting the capacity of the system by interference. Through a different approach $[1,2]$, we could consider a set of users being scheduled by the base station and improve the system capacity instead of limiting it. Scheduling strategies may follow two different approaches. The base station example is a centralized approach, given that there is a network element that schedules the different users in a coordinated way under some particular knowledge about the network state. Decentralized approaches would leverage the network burden given that a set of network elements decide their own scheduling strategies based on whatever information they have available. The decentralized approach intrinsically underperforms the centralized approach, however the information needed for the scheduling of the users maybe significantly lower.

In both cases, we need to set, for each of the users, a utility function to be maximized, knowing that the utility functions depends on the users transmission strategies or actions. Under a scheduling umbrella, a reasonable choice for the user utility function is any that depends on its achievable rate. Under a normalized noise, this is defined as

$$
R_{t}^{i}=\log \left(1+\frac{\left|h_{t}^{i}\right|^{2} u_{t}^{i}}{1+\sum_{j \neq i}\left|h_{t}^{j}\right|^{2} u_{t}^{j}}\right)
$$

where for user $i$ at time $t, h_{t}^{i}$ is the fading channel and $u_{t}^{i}$ is the power transmitted.

Decentralized scheduling strategies can be solved by means of the definition of a non cooperative game that would norm what resources should each user access. Decentralized scheduling strategies need to be provided, among others, in scenarios such as ad-hoc networks [3, 4], cognitive radio scenarios [5, 6], uplink [7] and distributed scenarios [8].

System evolution and finite resources lead in many cases to dynamic scenarios where for example the channel, batteries or even the utility function change with time. In these cases special attention should be given to the formulation of the game by means of a dynamic game and several results are already available in the literature for access methods [9], network selection [10] or packet delivery ratio [11].

In this work we aim to implement distributed scheduling in a changing environment and we will formulate the process as a competitive dynamic game. The approach we propose is quite novel compared to previous applications of dynamic games to wireless communications because we are able to include any kind of dynamics (channel varying characteristic, utility variation or system dynamics) to consider more realistic scenarios. Unfortunately, these games are quite complicated because each user has to solve the corresponding optimum control problem as best response to other users strategies in an iterative game. We propose to follow here a different approach and extend the concept of (static) potential games to include dynamics (dynamic potential game, DPG). With this aim we formulate an extended Euler equation in term of states and actions and relate the existence of an equivalent Multivariate Optimum Control Problem (MOCP) to the integrability or conservativeness of an associated vectorial field. This approach extends our previous work [12] by allowing system and utility function dynamics. The resulting non stationary problem is solved using an MOCP, augmenting the dimension of the state vector including time, to perform a discrete solution of the Bellman equation. 


\section{PROBLEM STATEMENT}

In a dynamic game we have a set of players $\mathcal{Q}=\{1 \ldots Q\}$, where for each player $i$ its utility function at discrete time $t$ is given by $\pi^{i}\left(\mathbf{x}_{t}, \mathbf{u}_{t}, t\right)$, and it depends both on the system state $\mathrm{x}_{t}=\left(x_{t}^{1} \ldots x_{t}^{i} \ldots x_{t}^{Q}\right)$, with $x_{t}^{i} \in \mathcal{X}^{i}$, and on the set of actions of all players, denoted in vector form as $\mathbf{u}_{t}=\left(u_{t}^{1} \ldots u_{t}^{i} \ldots u_{t}^{Q}\right)$ with $u_{t}^{i} \in \mathcal{U}^{i}$. It can be noticed that in the most general case, both the utility function and the system equation are time dependent. The discrete time dynamic game can be represented $\forall i$ as:

$$
\begin{aligned}
V^{i}\left(\mathbf{x}_{0}\right) & =\max _{\left\{u_{t}^{i}\right\}} \sum_{t=0}^{\infty} \beta^{t} \pi^{i}\left(\mathbf{x}_{t}, \mathbf{u}_{t}, t\right) \\
\text { s.t. }: \mathbf{x}_{t+1} & =f\left(\mathbf{x}_{t}, \mathbf{u}_{t}, t\right), g_{i}\left(\mathbf{x}_{t}, \mathbf{u}_{t}, t\right) \leq 0, \mathbf{x}_{0} \text { known }
\end{aligned}
$$

where $\mathbf{x}_{t+1}=f\left(\mathbf{x}_{t}, \mathbf{u}_{t}, t\right)$ is the system state equation with components $x_{t+1}^{i}=f^{i}\left(\mathbf{x}_{t}, \mathbf{u}_{t}, t\right)$ defining a Markovian model, parameter $\beta<1$ is the discount factor and some extra constraints are included $g_{i}\left(\mathbf{x}_{t}, \mathbf{u}_{t}, t\right) \leq 0$ because in most of the applications states and actions are constrained. Each user $i$ intends to find the optimum sequence of actions $\left\{u_{t}^{i}\right\}$ that maximizes its value function $V^{i}\left(\mathbf{x}_{0}\right)$ expressed in terms of its own current and future (discounted) utility function $\pi^{i}\left(\mathrm{x}_{t}, \mathbf{u}_{t}, t\right)$. Solving these problems requires finding the sequence of actions $\left\{\mathbf{u}_{t}^{*}\right\}$ that provides a Nash equilibrium, whose definition is similar as in static games:

$$
\sum_{t=0}^{\infty} \beta^{t} \pi^{i}\left(\mathbf{x}_{t}, u_{t}^{* i}, \mathbf{u}_{t}^{*-i}, t\right) \geq \sum_{t=0}^{\infty} \beta^{t} \pi^{i}\left(\mathbf{x}_{t}, u_{t}^{i}, \mathbf{u}_{t}^{*-i}, t\right) \forall i
$$

where the equation must hold $\forall u_{t}^{i} \in \mathcal{U} i$ and where $u_{t}^{* i} \in \mathcal{U}^{i}$ represents the optimum action of user $i$ at time $t$ and $\mathbf{u}_{t}^{*-i}$ is the same concept for all users except $i$.

The objective of this paper is to show that, under some hypotheses, the open loop game in (1) is in fact a DPG and can be solved by an equivalent non stationary MOCP

$$
\begin{gathered}
V\left(\mathbf{x}_{0}\right)=\max _{\left\{\mathbf{u}_{t}\right\}} \sum_{t=0}^{\infty} \beta^{t} \Pi\left(\mathbf{x}_{t}, \mathbf{u}_{t}, t\right) \\
\text { s.t }: \mathbf{x}_{t+1}=f\left(\mathbf{x}_{t}, \mathbf{u}_{t}, t\right), g_{i}\left(\mathbf{x}_{t}, \mathbf{u}_{t}, t\right) \leq 0 \quad \forall i, \mathbf{x}_{0} \text { known }
\end{gathered}
$$

giving some new insights about the existence and relationship between potential functions such as $\Pi\left(\mathbf{x}_{t}, \mathbf{u}_{t}, t\right)$ and conservative vector fields. To the best of our knowledge, our derivations follow a quite different path from previous results in the literature, providing very general results.

A reduced form for the system state equation in (1) is possible when the action set can be expressed in terms of the current and next states, i.e. $u_{t}^{i}=\zeta\left(\boldsymbol{x}_{t}, \boldsymbol{x}_{t+1}, \mathbf{u}_{t}^{-i}, t\right)$. In $[13,14]$ a rigorous description of the game in this reduced form is given and by means of the Euler equation (EE) the concept of dynamic potential game shows up as a reformulation of the original papers by Dechert [15]-[17]. However, under a general perspective, it may not be possible to find the reduced form and therefore, a more general theory is highly desirable. In [16], this scenario is considered and the conditions are presented from a Hamiltonian perspective. More recently, [18] shows rigorously the general procedure for the continuos time model also exploiting the Hamiltonian perspective. Our contribution follows a different approach starting with the proposal of a more general Euler equation (GEE) that is able to represent utility functions in terms of states and actions instead of the reduced form just in terms of states. In chapter 6 of [19] some hints about this formulation for the continuos time optimum control problem are given. Our second important contribution follows the conservative vector field framework established by Monderer/Shapley [20] and Slade [21] to define static potential games, to further generalize and extend the potential game scenario to a dynamic environment.

\subsection{Euler equation in general form: solving the game}

Following Sage's framework [19] (defined for control problems but also applicable to games) we define the Lagrangian of the value function in (1):

$$
\begin{aligned}
& \mathcal{L}^{i}\left(\mathbf{x}_{t}, \mathbf{u}_{t}, \lambda_{t}^{i i}, \lambda_{t}^{i j}, t\right)=\sum_{t=0}^{\infty} \beta^{t}\left(\pi^{i}\left(\mathbf{x}_{t}, \mathbf{u}_{t}, t\right)+\right. \\
& \left.\quad+\sum_{\forall j} \lambda_{t+1}^{i j}\left(-x_{t+1}^{j}+f^{j}\left(\mathbf{x}_{t}, \mathbf{u}_{t}, t\right)\right)\right)
\end{aligned}
$$

For simplicity, the constraints $g_{i}\left(\mathbf{x}_{t}, \mathbf{u}_{t}, t\right) \leq 0 \quad \forall i$ are not considered in this analysis as the game and the MOCP share the same constraint set. The general Euler equation is a set of two equations:

$$
\begin{gathered}
\frac{\partial \pi^{i}\left(\mathbf{x}_{t}, \mathbf{u}_{t}, t\right)}{\partial x_{t}^{i}}+\sum_{\forall j} \lambda_{t+1}^{i j} \frac{\partial f^{j}\left(\mathbf{x}_{t}, \mathbf{u}_{t}, t\right)}{\partial x_{t}^{i}}-\lambda_{t}^{i i}=0 \\
\frac{\partial \pi^{i}\left(\mathbf{x}_{t}, \mathbf{u}_{t}, t\right)}{\partial u_{t}^{i}}+\sum_{\forall j} \lambda_{t+1}^{i j} \frac{\partial f^{j}\left(\mathbf{x}_{t}, \mathbf{u}_{t}, t\right)}{\partial u_{t}^{i}}=0
\end{gathered}
$$

\subsection{Euler equation in general form: solving the MOCP}

If we proceed in a similar way analyzing the MOCP we have:

$$
\begin{aligned}
\mathcal{L}\left(\mathbf{x}_{t}, \mathbf{u}_{t}, \lambda_{t}^{i}, t\right) & =\sum_{t=0}^{\infty} \beta^{t}\left(\Pi\left(\mathbf{x}_{t}, \mathbf{u}_{t}, t\right)+\right. \\
& \left.+\sum_{\forall i} \lambda_{t+1}^{i}\left(-x_{t+1}^{i}+f^{i}\left(\mathbf{x}_{t}, \mathbf{u}_{t}, t\right)\right)\right)
\end{aligned}
$$

The general Euler equation is also a set of two equations that can be solved given:

$$
\begin{gathered}
\frac{\partial \Pi\left(\mathbf{x}_{t}, \mathbf{u}_{t}, t\right)}{\partial x_{t}^{i}}+\lambda_{t+1}^{i} \frac{f^{i}\left(\mathbf{x}_{t}, \mathbf{u}_{t}, t\right)}{\partial x_{t}^{i}}-\lambda_{t}^{i}=0 \\
\frac{\partial \Pi\left(\mathbf{x}_{t}, \mathbf{u}_{t}, t\right)}{\partial u_{t}^{i}}+\lambda_{t+1}^{i} \frac{f^{i}\left(\mathbf{x}_{t}, \mathbf{u}_{t}, t\right)}{\partial u_{t}^{i}}=0
\end{gathered}
$$




\subsection{Conservative vector field}

The concept of conservative vector field is the core of the analysis of games modeled as potential functions. It converts the game into a standard optimization problem in the static case and into an MOCP for the dynamic case game. Following the pioneering works of [20] and [21], the definition of a static game is given by

$$
\max _{u^{i} \in \mathcal{U}^{i}} \pi^{i}\left(u^{i}, \mathbf{u}^{-i}\right) \quad \forall i,
$$

the condition for this game to be of the potential is:

$$
\frac{\partial^{2} \pi^{i}(\mathbf{u})}{\partial u^{i} \partial u^{j}}=\frac{\partial^{2} \pi^{j}(\mathbf{u})}{\partial u^{i} \partial u^{j}} \quad \forall i, j
$$

and the potential function can be found by solving the line integral

$$
\Pi(\mathbf{u})=\sum_{i=1}^{Q} \int \frac{\partial \pi^{i}(\mathbf{u})}{\partial u^{i}} d u^{i}
$$

Roughly speaking, in $[20,21]$ it is stated that the condition of existence of a potential function is that the set of individual utility functions is in fact a conservative vector field $\vec{F}(\mathbf{u})=\left(\begin{array}{llll}\frac{\partial \pi^{1}}{\partial u^{1}} & \frac{\partial \pi^{2}}{\partial u^{2}} & \cdots & \frac{\partial \pi^{Q}}{\partial u^{Q}}\end{array}\right)$ and the existence of the potential function is related to the integrability of this field that is guaranteed as it is conservative. The extension to dynamic games is conceptually straightforward: instead of the optimization conditions (zero gradient) already stated we can apply optimality conditions in terms of the GEE and the potential function would lead to an equivalent MOCP.

\subsection{Equivalence between the game and the MOCP}

If we compare (2)-(3) with (4)-(5) we reach a preliminary conclusion: $\lambda_{t}^{i j}$ must be irrelevant in the optimization process. This is the first condition that Dragone [18] states, now confirmed by our analysis. Furthermore, we can establish that the equivalence between the dynamic game and the control problem is related to the existence of a conservative vector field such that the gradient of the potential function:

$$
\nabla \Pi\left(\mathbf{x}_{t}, \mathbf{u}_{t}, t\right)=\left(\begin{array}{cccccc}
\frac{\partial \pi^{1}}{\partial x_{t}^{1}} & \cdots & \frac{\partial \pi^{Q}}{\partial x_{t}^{Q}} & \frac{\partial \pi^{1}}{\partial u_{t}^{1}} & \cdots & \frac{\partial \pi^{Q}}{\partial u_{t}^{Q}}
\end{array}\right)
$$

The conditions for this existence were defined by Dechert in [16] and reproduced in [12]. The equivalent MOCP is obtained solving the corresponding line integral:

\subsection{Proportional Fair Scheduling}

Proportional Fair (PF) schemes provides a good balance between system throughput and fairness, and serves first to those users whose average achieved rates are below their requested rate. We propose a game under this same philosophy:

$$
\begin{array}{r}
\max _{\left\{u_{t}^{2}\right\}} \sum_{t=0}^{\infty} \beta^{t} x_{t}^{i} \quad \text { s.t. }: x_{t+1}^{i}=\left(1-\frac{1}{t}\right) x_{t}^{i}+\frac{1}{t} R_{t}^{i} \\
0 \leq u_{t}^{i} \leq \mathcal{U}_{\max }^{i}, \quad x_{0}^{i}=\mathcal{X}_{\text {ni }}^{i} \forall i
\end{array}
$$

where basically each user intends to maximize its average rate, that is the actual state $x_{t}^{i}$. Taking into account that different users have uncorrelated fading, it is expected a certain degree of fairness. It is straightforward to show that this game fulfills Dechert conditions $[16,12]$ and the equivalent MOCP

$$
\begin{aligned}
& \max _{\left\{\mathbf{u}_{t}\right\}} \sum_{t=0}^{\infty} \beta^{t} \sum_{i=1}^{Q} x_{t}^{i} \quad \text { s.t. }: x_{t+1}^{i}=\left(1-\frac{1}{t}\right) x_{t}^{i}+\frac{1}{t} R_{t}^{i} \text {, } \\
& 0 \leq u_{t}^{i} \leq \mathcal{U}_{\max }^{i}, \quad x_{0}^{i}=\mathcal{X}_{\text {ini }}^{i} \forall i
\end{aligned}
$$

\subsection{Equal-Rate scheduling algorithm}

Considering now that the actual state of user $i$ is the accumulated rate, we define a strategy where we try to keep the user rates as close as possible performing some kind of equal rate (ER) optimization:

$$
\begin{aligned}
& \max _{\left\{u_{t}^{i}\right\}} \sum_{t=0}^{\infty} \beta^{t}\left((1-\alpha) R_{t}^{i}+\alpha \sum_{j \neq i}\left(x_{t}^{i}-x_{t}^{j}\right)^{2}\right) \\
& \quad \text { s.t. }: x_{t+1}^{i}=x_{t}^{i}+R_{t}^{i}, 0 \leq u_{t}^{i} \leq \mathcal{U}_{\max }^{i}, \quad x_{0}^{i}=\mathcal{X}_{\text {ini }}^{i} \forall i
\end{aligned}
$$

where $\alpha$ weights different contributions according to the design priorities. The equivalent MOCP is given by

$$
\begin{aligned}
& \max _{\left\{\mathbf{u}_{t}\right\}} \sum_{t=0}^{\infty} \beta^{t}\left((1-\alpha) \log \left(1+\sum_{i=1}^{Q}\left|h_{t}^{i}\right|^{2} u_{t}^{i}\right)+\right. \\
& \left.+\alpha \sum_{i=1}^{Q} \sum_{j>i}\left(x_{t}^{i}-x_{t}^{j}\right)^{2}\right) \quad \text { s.t. }: x_{t+1}^{i}=x_{t}^{i}+R_{t}^{i} \\
& 0 \leq u_{t}^{i} \leq \mathcal{U}_{\max }^{i}, x_{0}^{i}=\mathcal{X}_{\text {ini }}^{i} \forall i
\end{aligned}
$$

$u_{t}^{i}$ where the first equivalence is show in [12] and the second is
straightforward.

\section{COMPUTATIONAL METHODS AND RESULTS}

There are two main difficulties to numerically solve the MOCP in (6) and (7) $i$ ) they are non stationary as they include time dependent parameters and also ii) the users operate in a state space and have utility functions that are continuous. However, we can overcome the non stationary issue by augmenting the state space to include the time as a new feature 
of the state [22]. We define a state $\mathbf{x}$ that belongs to the state space $\mathcal{X}=\left(\mathcal{X}^{1} \ldots \mathcal{X}^{i} \ldots \mathcal{X}^{Q}\right)$. The augmented state space becomes $\widetilde{\mathcal{X}}=(\mathcal{X}, \mathcal{K})$, where $\mathcal{K}$ denotes the set of nonnegative integers, and $\widetilde{\mathbf{x}}=(\mathbf{x}, t)=\left(x^{1} \ldots x^{i} \ldots x^{Q}, t\right) \in \widetilde{\mathcal{X}}$ is the augmented state.

Now, if we assume that the cost per stage is bounded, $|\Pi(\widetilde{\mathbf{x}}, \mathbf{u})| \leq M$, for all $(\widetilde{\mathrm{x}}, \mathbf{u}) \in \widetilde{\mathcal{X}} \times \mathcal{U}$ with $\mathcal{U}=$ $\left(\mathcal{U}^{1} \ldots \mathcal{U}^{i} \ldots \mathcal{U}^{Q}\right)$ and some scalar $M$, and the discount factor satisfies $0<\beta<1$, then we can use Dynamic Programming (DP) methods [22] to find the optimal actions for these problems. For that we reformulate the MOCP using the Bellman equation for every possible state:

$$
V(\widetilde{\mathbf{x}})=\max _{\mathbf{u} \in \mathcal{U}} \Pi(\widetilde{\mathbf{x}}, \mathbf{u})+\beta V(f(\widetilde{\mathbf{x}}, \mathbf{u}))
$$

where it should be noted that now both $\Pi(\widetilde{\mathbf{x}}, \mathbf{u})$ and $f(\widetilde{\mathbf{x}}, \mathbf{u})$ are defined over the extended state space. Still, if the state space and the utility function are continuous, then DP methods becomes impractical. Two common approaches for dealing with continuous state spaces are: to define an appropriate grid on the continuous space or to find a parametric approximation of the value function $V(\widetilde{\mathbf{x}})$. Given that $V(\widetilde{\mathbf{x}})$ is not available, the grid approach is more effective. Thus we sample the state space with cardinality $S$, and then apply a specific DP method to numerically solve the MOCP. We choose the Value Iteration (VI) algorithm for its reduced complexity per iteration, which is especially relevant when the state-grid has fine resolution. In the algorithm we define the operator $[\cdot]$ as the closest space point.

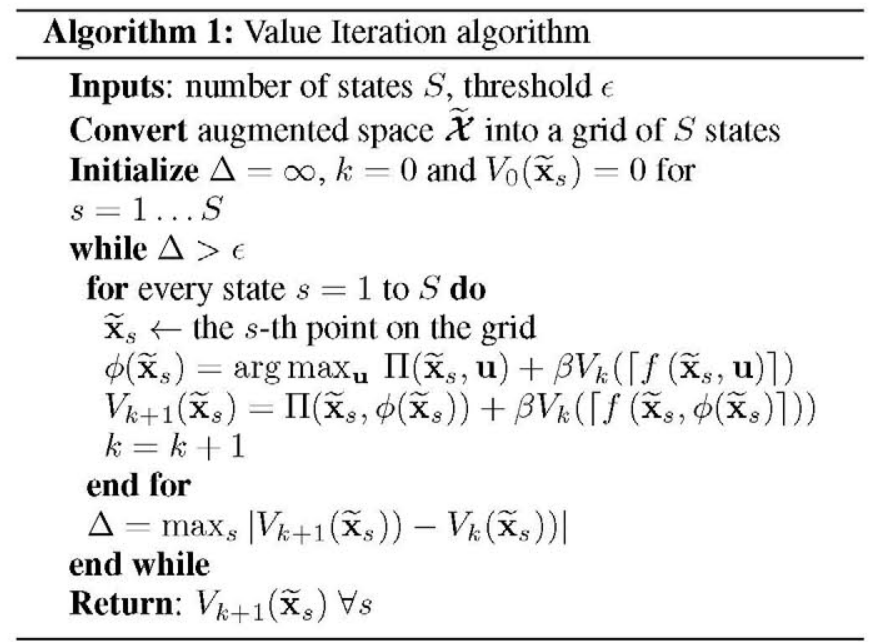

For illustrative purposes, we simulate a simple scenario with $Q=2$ users. A correlated fading scenario is modeled by means of sinusoids with different frequency and amplitude for each user (see Fig. 1). The maximum transmitted power is $U_{\max }^{i}=5 \forall i$, with 20 possible power levels per user, which amounts to 400 possible actions. The discount factor is $\beta=0.9$. We discretize the rate into a grid of 30 points per user. The non stationarity of the environment is surmounted by augmenting the state space with $T=20$ time

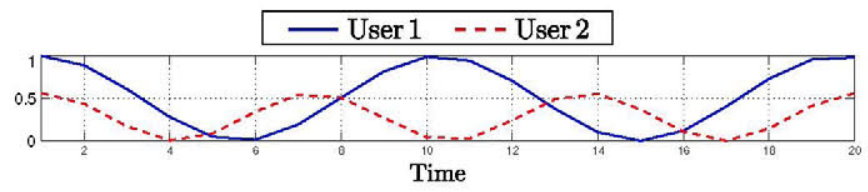

Fig. 1. Channel coefficients $\left|h_{t}^{i}\right|^{2}$.

steps. Hence, the augmented state space has $S=20^{2} \times 20=$ 8000 states. We run the VI algorithm over the two scheduling schemes given in (6) and (7). The numerical experiments illustrate how the control framework can efficiently solve the complicated dynamical game theoretic formulation. For the equal-rate problem, the utility function uses $\alpha=0.9$.

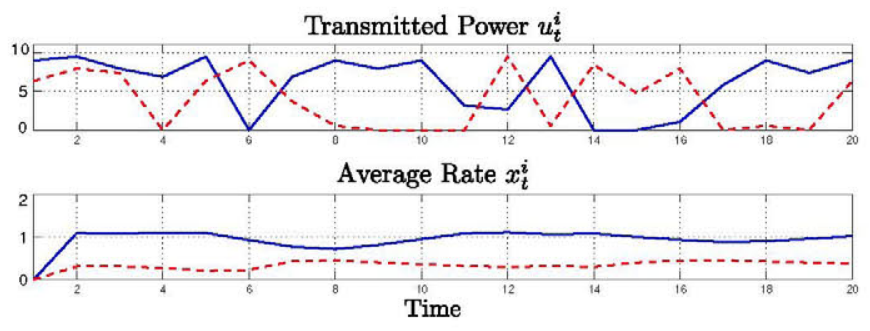

Fig. 2. Proportional fair.

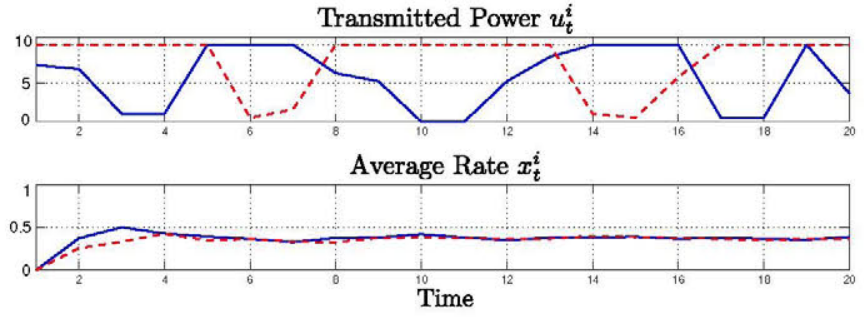

Fig. 3. Equal rate.

The solution of the PF game leads to an efficient solution (see Fig. 2), in which both users try to minimize interference and, hence, they approach their maximum rates. In Fig. 3, we observe that the agents achieve very similar rates. The trend is that the user with a channel with less average gain (User 2) tries to achieve its maximum rate, while the user with higher average gain channel (User 1) reduces its transmitting power to match the rate of user 2 . In other words, the user with poorest channel sets a bottleneck for the other user.

\section{CONCLUSIONS}

In this work a general framework for solving a DPG that norm a decentralizing scheduling algorithm is proposed. Additional degrees of time-dependability are included, taking into account both, possible time variations of the utility function and also non stationarity of the system state function. The proposed scheduling games are shown to turn into an MOCP that is effectively solved by means of a Value Iteration algorithm. 


\section{REFERENCES}

[1] A. Lozano, "Enhancing UMTS High-Speed Downlink Packet Access with Dual-Antenna Terminals," in IEEE Global Telecommunications Conference (GLOBECOM'02), Nov. 2002, vol. 21, pp. 732-736.

[2] P. Viswanath, D. M. C. Tse, and R. Laroia, "Opportunistic Beamforming Using Dumb Antennas," IEEE Transactions on Information Theory, vol. 48, no. 6, pp. 1277-1294, June 2002.

[3] Yongkang Xiao, Xiuming Shan, and Yong Ren, "Game theory models for IEEE 802.11 DCF in wireless ad hoc networks," IEEE Communications Magazine, vol. 43, no. 3, pp. S22-S26, March 2005.

[4] V. Srivastava, J. Neel, AB. MacKenzie, R. Menon, L.A Dasilva, J.E. Hicks, J.H. Reed, and R.P. Gilles, "Using game theory to analyze wireless ad hoc networks," IEEE Communications Surveys Tutorials, vol. 7, no. 4, pp. 4656, Fourth 2005.

[5] Zhu Ji and K.J.R. Liu, "Cognitive radios for dynamic spectrum access - dynamic spectrum sharing: A game theoretical overview," IEEE Communications Magazine, vol. 45, no. 5, pp. 88-94, May 2007.

[6] D.B. Rawat and S. Shetty, "Game theoretic approach to dynamic spectrum access with multi-radio and QoS requirements," in IEEE Global Conference on Signal and Information Processing (GlobalSIP), Dec 2013, pp. 1150-1153.

[7] G. Scutari, S. Barbarossa, and D.P. Palomar, "Potential games: A framework for vector power control problems with coupled constraints," in ICASSP 2006 Proceedings, 2006, vol. 4, pp. IV-IV.

[8] Hui Zhou, Pingyi Fan, and Jie Li, "Global proportional fair scheduling for networks with multiple base stations," Vehicular Technology, IEEE Transactions on, vol. 60, no. 4, pp. 1867-1879, May 2011.

[9] Guopeng Zhang and Hailin Zhang, "Modelling ieee $802.11 \mathrm{dcf}$ in wireless lans as a dynamic game with incompletely information," in Wireless, Mobile and Multimedia Networks, 2008. IET International Conference on, Jan 2008, pp. 215-218.

[10] D. Niyato and E. Hossain, "Dynamics of network selection in heterogeneous wireless networks: An evolutionary game approach," IEEE Transactions on Vehicular Technology, vol. 58, no. 4, pp. 2008-2017, May 2009.

[11] D.B. Smith, M. Portmann, Wee Lum Tan, and W. Tushar, "Multi-source-destination distributed wireless networks: Pareto-efficient dynamic power control game with rapid convergence," IEEE Transactions on Vehicular Technology, vol. 63, no. 6, pp. 2744-2754, July 2014.
[12] S. Zazo, J. Zazo, and M. Sánchez-Fernández, "A control theoretic approach to solve a constrained uplink power dynamic game," in Proceeding of the European Signal Processing Conference, EUSIPCO 2014, 2014.

[13] O. Hernández-Lerma and D. González-Sánchez, Discrete Time Stochastic Control and Dynamic Potential Games: The Euler Equation Approach, Springer International Publishing, Aug. 2013.

[14] D. González-Sánchez and O. Hernández-Lerma, "Dynamic potential games: The discrete-time stochastic case," Dynamic Games and Applications, vol. 4, no. 3, pp. 309-328, 2014.

[15] W. D. Dechert, "Optimal control problems from secondorder difference equations," Journal of Economic Theory, vol. 19, no. 1, pp. 50-63, Oct. 1978.

[16] W. D. Dechert, "Non cooperative dynamic games: a control theoretic approach," Unpublished. Available on request to the author, 1997.

[17] W. D. Dechert and William A. Brock, "The lake game," Economic and Ecological Modelling, The Beijer Institute, The Royal Academy of Sciences, , no. 24, 2000.

[18] D. Dragone, L. Lambertini, G. Leitmann, and A. Palestini, "Hamiltonian potential functions for differential games," Proceedings of IFAC CAO, vol. 9, 2009.

[19] Andrew P. Sage and Chelsea C. White, Optimum systems control, Englewood Cliffs, N.J. : Prentice-Hall, 2d ed edition, 1977.

[20] D. Monderer and L. S. Shapley, "Potential games," Games and Economic Behavior, vol. 14, no. 1, pp. 124 $-143,1996$.

[21] M. E. Slade, "What does an oligopoly maximize?", The Journal of Industrial Economics, vol. 42, no. 1, pp. pp. 45-61, 1994.

[22] D. P. Bertsekas, Dynamic Programming and Optimal Control, Athena Scientific, 2012. 